\title{
A PROJECTION FORMULA FOR THE ASKEY-WILSON POLYNOMIALS AND AN APPLICATION
}

\author{
MIZAN RAHMAN
}

(Communicated by Kenneth R. Meyer)

\begin{abstract}
A projection formula for $p_{n}(x ; a, b, c, d \mid q)$, the Askey-Wilson polynomials, is obtained by using a generalization of Askey and Wilson's $q$-beta integral. The result is used to find a $q$-analogue of the Feldheim-Vilenkin formula for ultraspherical polynomials. A $q$-analogue of the ultraspherical polynomials, other than the one due to Rogers, is also introduced.
\end{abstract}

1. Introduction. The main objective of this paper is to find a $q$-analogue of the projection formula

$\frac{(1-x)^{\alpha+\mu}}{(1+x)^{n+\alpha+1}} \frac{P_{n}^{(\alpha+\mu, \beta)}(x)}{P_{n}^{(\alpha+\mu, \beta)}(1)}=\frac{2^{\mu} \Gamma(\alpha+\mu+1)}{\Gamma(\alpha+1) \Gamma(\mu)} \int_{x}^{1} \frac{(1-y)^{\alpha}(y-x)^{\mu-1}}{(1+y)^{n+\alpha+\mu+1}} \frac{P_{n}^{(\alpha, \beta)}(y)}{P_{n}^{(\alpha, \beta)}(1)} d y$ $-1<x<1, \alpha>-1, \mu>0$, where $P_{n}^{(\alpha, \beta)}(x)$ is the Jacobi polynomial defined by

$$
P_{n}^{(\alpha, \beta)}(x)=\frac{(\alpha+1)_{n}}{n !}{ }_{2} F_{1}\left(-n, n+\alpha+\beta+1 ; \alpha+1 ; \frac{1-x}{2}\right) .
$$

A change of variable on both sides of (1.1) gives the accompanying formula

$$
\frac{(1+x)^{\beta+\mu}}{(1-x)^{n+\beta+1}} \frac{P_{n}^{(\alpha, \beta+\mu)}(x)}{P_{n}^{(\beta+\mu, \alpha)}(1)}=\frac{2^{\mu} \Gamma(\beta+\mu+1)}{\Gamma(\beta+1) \Gamma(\mu)} \int_{-1}^{x} \frac{(1+y)^{\beta}(x-y)^{\mu-1}}{(1-y)^{n+\beta+\mu+1}} \frac{P_{n}^{(\alpha, \beta)}(y)}{P_{n}^{(\beta, \alpha)}(1)} d y
$$

$-1<x<1, \beta>-1, \mu>0$. Askey and Fitch [3] used (1.1) to give a simple proof of Feldheim [10] and Vilenkin's [23] formula

$$
\begin{aligned}
\frac{C_{n}^{\nu}(\cos \theta)}{C_{n}^{\nu}(1)}= & \frac{2 \Gamma(\nu+1 / 2)}{\Gamma(\lambda+1 / 2) \Gamma(\nu-\lambda)} \int_{0}^{\pi / 2} \sin ^{2 \lambda} \phi \cos ^{2 \nu-2 \lambda-1} \phi\left(1-\sin ^{2} \theta \cos ^{2} \phi\right)^{n / 2} \\
& \cdot \frac{C_{n}^{\lambda}\left(\cos \theta\left(1-\sin ^{2} \theta \cos ^{2} \phi\right)^{-1 / 2}\right)}{C_{n}^{\lambda}(1)} d \phi, \quad \nu>\lambda>-\frac{1}{2}
\end{aligned}
$$

where

$$
\begin{aligned}
C_{n}^{\lambda}(\cos \theta) & =\frac{(2 \lambda)_{n}}{(\lambda+1 / 2)_{n}} P_{n}^{(\lambda-1 / 2, \lambda-1 / 2)}(\cos \theta) \\
& =\sum_{k=0}^{n} \frac{(\lambda)_{k}(\lambda)_{n-k}}{k !(n-k) !} \cos (n-2 k) \theta
\end{aligned}
$$

Received by the editors May 7, 1987 and, in revised form, June 5, 1987.

1980 Mathematics Subject Classification (1985 Revision). Primary 33A65, 33A70.

Key words and phrases. q-ultraspherical and Askey-Wilson polynomials, Feldheim-Vilenkin formula, balanced and very-well-poised basic hypergeometric series.

This work was supported in part by NSERC grant \#A6197. 
is the ultraspherical polynomial; see Szegö [22]. Feldheim [10] used (1.4) and the positivity of Fejér's [9] sum $\sum_{k=0}^{n} P_{k}(x)$ for Legendre polynomials to show that

$$
\sum_{k=0}^{n} \frac{C_{k}^{\nu}(\cos \theta)}{C_{k}^{\nu}(1)}>0, \quad 0 \leq \theta<\pi, \nu \geq \frac{1}{2} .
$$

Askey and Fitch [3] used (1.4) to deduce the integral representation

$$
\frac{P_{n}^{(\alpha, \alpha)}(x)}{P_{n}^{(\alpha, \alpha)}(1)}=\int_{-1}^{1} \frac{P_{n}^{(\beta, \beta)}(y)}{P_{n}^{(\beta, \beta)}(1)} d \mu(y)
$$

where $\alpha>\beta \geq-1 / 2,-1 \leq x \leq 1$, and $d \mu(y)$ is a positive measure which depends on $x$ but not on $n$.

Askey [2] also gave an elementary proof of Koornwinder's [12, 13] Laplace-type integral representation for Jacobi polynomials by using (1.1). Laine [15] found an extension of (1.1) to obtain a formula that is equivalent to Koornwinder's $[12,14]$ addition formula for Jacobi polynomials.

To find a $q$-analogue of (1.1) we shall need the following extension of the betaintegral

$$
\begin{gathered}
\int_{0}^{\pi}\left\{\left(e^{2 i \theta}, e^{-2 i \theta} ; q\right)_{\infty} h\left(\cos \theta, a_{1} a_{2} a_{3} a_{4} a_{5}\right) / \prod_{j=1}^{5} h\left(\cos \theta, a_{j}\right)\right\} d \theta \\
\quad=2 \pi \prod_{j=1}^{5}\left(a_{1} a_{2} a_{3} a_{4} a_{5} / a_{j} ; q\right)_{\infty} /(q ; q)_{\infty} \prod_{1 \leq j<k \leq 5}\left(a_{j} a_{k} ; q\right)_{\infty},
\end{gathered}
$$

where

$$
(A ; q)_{\infty}=\prod_{j=0}^{\infty}\left(1-A q^{j}\right), \quad 0<q<1
$$

$$
h(\cos \theta, a)=\prod_{j=0}^{\infty}\left(1-2 a \cos \theta q^{j}+a^{2} q^{2 j}\right)=\left(a e^{i \theta} ; q\right)_{\infty}\left(a e^{-i \theta} ; q\right)_{\infty}
$$

and $\max \left(\left|a_{j}\right|\right)<1, j=1, \ldots, 5$. If we set $a_{5}=0$ then (1.8) reduces to the $q$-beta integral of Askey and Wilson [6, (2.1)]. Formula (1.8) was given in Rahman [17].

Askey and Wilson [6] introduced the polynomials

$$
\begin{aligned}
& p_{n}(x)=p_{n}(x ; a, b, c, d \mid q)
\end{aligned}
$$

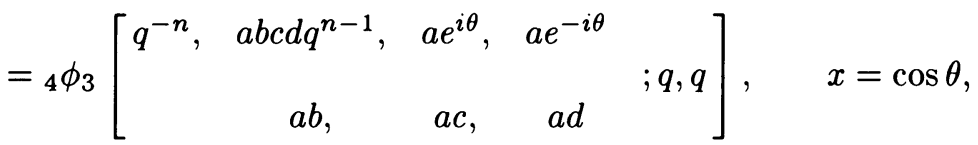

as a generalization of the Jacobi polynomials and showed that they are orthogonal on $[-1,1]$ with respect to the weight function

$$
\begin{aligned}
W(x) & \equiv W(x ; a, b, c, d \mid q) \\
& =\frac{h(x, 1) h(x,-1) h\left(x, q^{1 / 2}\right) h\left(x,-q^{1 / 2}\right)}{h(x, a) h(x, b) h(x, c) h(x, d)}\left(1-x^{2}\right)^{-1 / 2}
\end{aligned}
$$


where $a, b, c, d$ are either real or, if complex, occur in conjugate pairs such that their absolute values are less than 1 . We shall assume throughout this paper that $0<q<1$. The symbol on the right side of (1.11) represents a basic hypergeometric series defined by

$$
r+1 \phi_{r}\left[\begin{array}{c}
a_{1}, \quad a_{2}, \ldots, a_{r+1} \\
b_{1}, \ldots, b_{r}
\end{array} ; q, z\right]=\sum_{n=0}^{\infty} \frac{\left(a_{1}, a_{2}, \ldots, a_{r+1} ; q\right)_{n}}{\left(q, b_{1}, \ldots, b_{r} ; q\right)_{n}} z^{n}
$$

where

$$
\begin{aligned}
& \left(A_{1}, A_{2}, \ldots, A_{m} ; q\right)_{n}=\prod_{j=1}^{m}\left(A_{j} ; q\right)_{n}, \\
& (A ; q)_{n}=\left\{\begin{array}{l}
1, \quad n=0, \\
(1-A)(1-A q) \cdots\left(1-A q^{n-1}\right), \quad n=1,2, \ldots
\end{array}\right.
\end{aligned}
$$

If any one of the parameters $a_{1}, \ldots, a_{r+1}$ is of the form $q^{-k}, k$ a nonnegative integer, then the series in (1.13) terminates, otherwise we require that $|z|<1$ to ensure convergence. If $z=q$ and $b_{1} \cdots b_{r}=q a_{1} \cdots a_{r+1}$, then the ${ }_{r+1} \phi_{r}$ series is said to be balanced. If $q a_{1} \neq a_{2} b_{1}=a_{3} b_{2}=\cdots=a_{r+1} b_{r}$ then the series is called a nearly-poised series of the first kind, and it is a nearly-poised series of the second kind if $q a_{1}=a_{2} b_{1}=\cdots \neq a_{r+1} b_{r}$. The ${ }_{r+1} \phi_{r}$ series is called well-poised if $q a_{1}=a_{2} b_{1}=\cdots=a_{r+1} b_{r} ;$ if, in addition, $a_{2}=-a_{3}=q a_{1}^{1 / 2}$ then we call the series very-well-poised. Note that the ${ }_{4} \phi_{3}$ series in (1.11) is balanced and terminating.

Since there are four parameters in $p_{n}(x ; a, b, c, d \mid q)$ there are many ways to choose them so that a continuous $q$-Jacobi polynomial can be defined in the sense that $p_{n}(x)$ gives $P_{n}^{(\alpha, \beta)}(x)$ in the limit $q \rightarrow 1$. Askey and Wilson [6] took

$$
a=q^{(2 \alpha+1) / 4}, \quad b=q^{(2 \alpha+3) / 4}, \quad c=-q^{(2 \beta+1) / 4}, \quad d=-q^{(2 \beta+3) / 4}
$$

to define

$$
P_{n}^{(\alpha, \beta)}(x \mid q)=\frac{\left(q^{\alpha+1} ; q\right)_{n}}{(q ; q)_{n}}{ }_{4} \phi_{3}\left[\begin{array}{ccccc}
q^{-n}, & q^{n+\alpha+\beta+1}, & q^{(2 \alpha+1) / 4} e^{i \theta}, & q^{(2 \alpha+1) / 4} e^{-i \theta} & \\
& q^{\alpha+1}, & -q^{(\alpha+\beta+1) / 2}, & -q^{(\alpha+\beta+2) / 2}
\end{array}\right]
$$

as a $q$-analogue of the Jacobi polynomials. The author [18] chose

$$
a=q^{1 / 2}, \quad b=q^{\alpha+1 / 2}, \quad c=-q^{\beta+1 / 2}, \quad d=-q^{1 / 2}
$$

to introduce

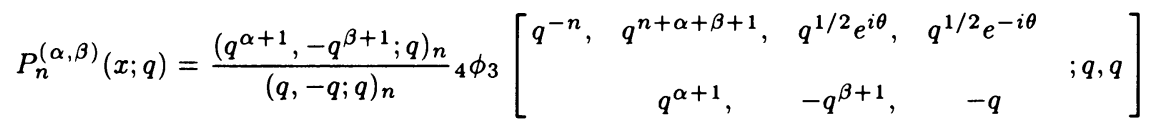

as another $q$-analogue. It is easy to see that

$$
\lim _{q \rightarrow 1} P_{n}^{(\alpha, \beta)}(x \mid q)=\lim _{q \rightarrow 1} P_{n}^{(\alpha, \beta)}(x ; q)=P_{n}^{(\alpha, \beta)}(x) .
$$

Also, as Askey and Wilson [6] pointed out, these analogoues are related by the formula

$$
P_{n}^{(\alpha, \beta)}\left(x \mid q^{2}\right)=\frac{(-q ; q)_{n}}{\left(-q^{\alpha+\beta+1} ; q\right)_{n}} q^{\alpha n} P_{n}^{(\alpha, \beta)}(x ; q)
$$


A number of important results for Jacobi polynomials have already been extended by using one or the other of these $q$-extensions (see, for example, Askey and Wilson [6], Gasper and Rahman [11], Rahman [19]), including a projection formula for $P_{n}^{(\alpha, \beta)}(x \mid q)$; see Nassrallah and Rahman [16]. However, we were unable to derive a $q$-analogue of (1.1) by choosing the parameters as in (1.15) or (1.17). So we were forced to consider other possibilities and we found that a projection formula analogous to (1.1) exists if we take

$$
a=q^{(2 \alpha+1) / 4}, \quad b=q^{(2 \alpha+3) / 4}, \quad c=-q^{(2 \alpha+1) / 4}, \quad d=-q^{\beta+(3-2 \alpha) / 4} .
$$

The basic formula that we shall prove in $\S 2$ is

$$
\begin{gathered}
\int_{-1}^{1} v\left(y ; a, b, c, \mu e^{i \theta}, \mu e^{-i \theta} \mid q\right) \frac{\left(a b c \mu e^{i \theta}, a b c \mu e^{-i \theta} ; q\right)_{n}}{\left(a b c \mu^{2} e^{i \phi}, a b c \mu^{2} e^{-i \phi} ; q\right)_{n}} p_{n}(y ; a, b, c, d \mid q) d y \\
=g\left(a, b, c, \mu e^{i \theta}, \mu e^{-i \theta} \mid q\right) \frac{(b c ; q)_{n}}{\left(b c \mu^{2} ; q\right)_{n}} p_{n}\left(x ; a \mu, b \mu, c \mu, d \mu^{-1} \mid q\right)
\end{gathered}
$$

where $x=\cos \theta, y=\cos \phi$ and

$$
\begin{aligned}
g\left(a_{1}, a_{2}, a_{3}, a_{4}, a_{5} \mid q\right) & =\int_{-1}^{1} v\left(y ; a_{1}, a_{2}, a_{3}, a_{4}, a_{5} \mid q\right) d y \\
& =2 \pi \prod_{j=1}^{5}\left(\frac{a_{1} a_{2} a_{3} a_{4} a_{5}}{a_{j}} ; q\right)_{\infty} /(q ; q)_{\infty} \prod_{1 \leq j<k \leq 5}\left(a_{j} a_{k} ; q\right)_{\infty},
\end{aligned}
$$

by (1.8). In (1.22) it is assumed that $-1<a, b, c<1$ and $0<\mu<1$. It is not hard to see that (1.22) is indeed a $q$-analogue of (1.1) if we choose the parameters as given in (1.21).

In $\S 3$ we shall derive the following $q$-analogue of the ultraspherical polynomials (1.25)

$$
\begin{aligned}
& G_{n}(\cos \theta ; \beta \mid q) \\
& =\frac{\left(-\beta^{1 / 2} q^{(n+1) / 2} e^{-2 i \theta},-\beta^{1 / 2} q^{(1-n) / 2} e^{2 i \theta} ; q\right)_{\infty}}{\left(-\beta^{1 / 2} q^{(n+1) / 2-[n / 2]} e^{-2 i \theta},-\beta^{1 / 2} q^{(1-n) / 2+[n / 2]} e^{2 i \theta} ; q\right)_{\infty}} e^{2 i(n / 2-[n / 2]) \theta} \\
& \frac{(\beta ; q)_{n}}{(q ; q)_{n}}{ }_{3} \phi_{2}\left[\begin{array}{cccc}
q^{-n}, & \beta, & -\beta^{-1 / 2} q^{(1-n) / 2} e^{-2 i \theta} & \\
& \beta^{-1} q^{1-n}, & -\beta^{1 / 2} q^{(1-n) / 2} e^{2 i \theta} & ; q, q
\end{array}\right]
\end{aligned}
$$

which is quite different and much more complicated than the $q$-ultraspherical polynomials of Rogers (see Askey and Ismail [4]). Formulas (3.5) and (3.7) will show, respectively, that $G_{n}(\cos \theta ; \beta \mid q)$ is a polynomial when $n$ is even, but not so when $n$ is odd unless it is divided by $e^{i \theta}+\beta^{1 / 2} e^{-i \theta}$. However, it has the property that

$$
\lim _{q \rightarrow 1} G_{n}\left(\cos \theta ; q^{\lambda} \mid q\right)=C_{n}^{\lambda}(\cos \theta), \quad \lambda>0
$$

and that there is a $q$-analogue of the Feldheim-Vilenkin formula (1.4) for $G_{n}(\cos \theta ; \beta \mid q)$, which we will derive in $\S 4$. 
2. Proof of (1.22). Let $j, n$ be nonnegative integers with $j \leq n$ and let $a, b, \mu, \nu$ be complex numbers with modulus less than 1 . Also let $0 \leq \theta \leq \pi$. Then, by (1.8) and (1.24)

$$
\begin{aligned}
\int_{-1}^{1} v\left(y ; a, b, \mu e^{i \theta}, \mu e^{-i \theta}, \nu \mid q\right) \frac{\left(a e^{i \phi}, a e^{-i \phi} ; q\right)_{j}\left(b e^{i \phi}, b e^{-i \phi} ; q\right)_{n-j}}{\left(a b \nu \mu^{2} e^{i \phi}, a b \nu \mu^{2} e^{-i \phi} ; q\right)_{n}} d y \\
=\int_{-1}^{1} v\left(y ; a q^{j}, b q^{n-j}, \mu e^{i \theta}, \mu e^{-i \theta}, \nu \mid q\right) \\
=g\left(a q^{j}, b q^{n-j}, \mu e^{i \theta}, \mu e^{-i \theta}, \nu \mid q\right) \\
=g\left(a, b, \mu e^{i \theta}, \mu e^{-i \theta}, \nu \mid q\right) q^{j(j+1)-2 n j} b^{-2 j} \\
\\
\cdot \frac{\left(a b, b \nu, b \mu e^{i \theta}, b \mu e^{-i \theta} ; q\right)_{n}\left(a \nu, q^{1-n} / b \nu \mu^{2}, a \mu e^{i \theta}, a \mu e^{-i \theta} ; q\right)_{j}}{\left(a b \mu^{2}, b \nu \mu^{2}, a b \mu \nu e^{i \theta}, a b \mu \nu e^{-i \theta} ; q\right)_{n}\left(q^{1-n} / b \nu, a \nu \mu^{2}, q^{1-n} e^{-i \theta} / b \mu, q^{1-n} e^{i \theta} / b \mu ; q\right)_{j}}
\end{aligned}
$$

Since, by Watson's formula [7, $8.5(2)]$,

$$
\begin{aligned}
& { }_{4} \phi_{3}\left[\begin{array}{cccc}
q^{-n}, & a b c d q^{n-1}, & a e^{i \phi}, & a e^{-i \phi} \\
a b, & a c, & a d
\end{array}\right] \\
& =\frac{\left(b e^{i \theta}, b e^{-i \theta} ; q\right)_{n}}{(a b, b / a ; q)_{n}}{ }_{8} W_{7}\left(a q^{-n} / b ; a e^{i \theta}, a e^{-i \theta}, q^{1-n} / b c, q^{1-n} / b d, q^{-n} ; q, c d q^{n}\right) \\
& =\sum_{j=0}^{n} \frac{\left(a q^{-n} / b, q^{1-n} / b c, q^{1-n} / b d, q^{-n} ; q\right)_{j}\left(1-a q^{2 j-n} / b\right)}{(q, a c, a d, a q / b ; q)_{j}\left(1-a q^{-n} / b\right)}\left(b^{2} c d\right)^{j} q^{3 n j-j(j+1)} \\
& \frac{\left(a e^{i \phi}, a e^{-i \phi} ; q\right)_{j}\left(b e^{i \phi}, b e^{-i \phi} ; q\right)_{n-j}}{(a b, b / a ; q)_{n}}
\end{aligned}
$$

where

$$
\begin{aligned}
& { }_{r+3} W_{r+2}\left(a ; q_{1}, \ldots, a_{r} ; q, z\right)
\end{aligned}
$$

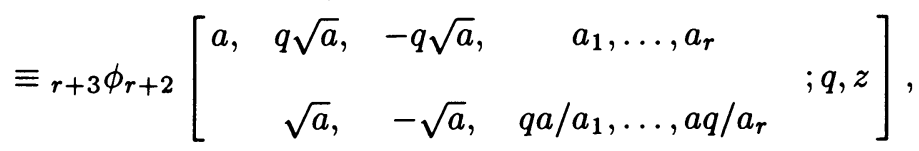

we find that

$$
\begin{aligned}
\int_{-1}^{1} v\left(y ; a, b, \mu e^{i \theta}, \mu e^{-i \theta}, \nu \mid q\right) & \frac{p_{n}(y ; a, b, c, d \mid q)}{\left(a b \nu \mu^{2} e^{i \phi}, a b \nu \mu^{2} e^{-i \phi} ; q\right)_{n}} d y \\
= & g\left(a, b, \mu e^{i \theta}, \mu e^{-i \theta}, \nu \mid q\right) \frac{\left(b \nu, b \mu e^{i \theta}, b \mu e^{-i \theta} ; q\right)_{n}}{\left(b / a, a b \mu^{2}, b \nu \mu^{2}, a b \mu \nu e^{i \theta}, a b \mu \nu e^{-i \theta} ; q\right)_{n}} \\
& \cdot{ }_{10} W_{9}\left(a q^{-n} / b ; a \mu e^{i \theta}, a \mu e^{-i \theta}, a \nu, q^{1-n} / b c, q^{1-n} / b d, q^{1-n} / b \nu \mu^{2}, q^{-n} ; q, c d q^{n}\right) .
\end{aligned}
$$

If we choose $\nu=c$ then the ${ }_{10} W_{9}$ series on the right side becomes an ${ }_{8} \phi_{7}$ series which, in turn, is expressible as $p_{n}(x)$ with $a, b, c, d$ replaced by $a \mu, b \mu, c \mu, d \mu^{-1}$, respectively. This completes the proof of (1.22). In order that (1.22) be seen as a $q$-analogue of (1.1) we need to impose the additional restrictions mentioned earlier, i.e., $-1<a, b, c<1$ and $0<\mu<1$. 
Transforming the ${ }_{4} \phi_{3}$ series in (1.11) by Sears' transformation formula [20, (8.3)] and interchanging the parameters we obtain from (1.22) another formula

$$
\begin{aligned}
& \int_{-1}^{1} v\left(y ; b, c, d, \mu e^{i \theta}, \mu e^{-i \theta} \mid q\right) \frac{\left(b c d \mu e^{i \theta}, b c d \mu e^{-i \theta} ; q\right)_{n}}{\left(b c d \mu^{2} e^{i \phi}, b c d \mu^{2} e^{-i \phi} ; q\right)_{n}} p_{n}(y ; a, b, c, d \mid q) d y \\
& \quad=g\left(b, c, d, \mu e^{i \theta}, \mu e^{-i \theta} \mid q\right) \frac{(b c, b d, c d ; q)_{n}}{\left(b c \mu^{2}, b d \mu^{2}, c d \mu^{2} ; q\right)_{n}} p_{n}\left(x ; a \mu^{-1}, b \mu, c \mu, d \mu \mid q\right)
\end{aligned}
$$

which can be seen as a $q$-analogue of (1.3) if we specialize the parameters as

$$
a=q^{\alpha+(3-2 \beta) / 4}, \quad b=q^{(2 \beta+1) / 4}, \quad c=-q^{(2 \beta+1) / 4}, \quad d=-q^{(2 \beta+3) / 4} .
$$

3. A $q$-analogue of the ultraspherical polynomials. By (1.11) and [7, $8.5(2)]$,

$$
\begin{aligned}
p_{n}\left(x ; a, a q^{1 / 2},-a,-q^{1 / 2} / a \mid q\right)={ }_{4} \phi_{3}\left[\begin{array}{llll}
q^{-n}, & a^{2} q^{n}, & a e^{i \theta}, & a e^{-i \theta} \\
& a^{2} q^{1 / 2}, & -a^{2}, & -q^{1 / 2}
\end{array}\right] \\
=\frac{\left(-a q^{1 / 2-n} e^{i \theta},-a q^{1 / 2-n} e^{-i \theta} ; q\right)_{n}}{\left(-a^{2} q^{1 / 2-n},-q^{1 / 2-n} ; q\right)_{n}} \\
{ }_{{ }_{8} W_{7}\left(-a^{2} q^{-n-1 / 2} ; a e^{i \theta}, a e^{-i \theta}, q^{1 / 2-n},-q^{-n}, q^{-n} ; q,-a^{2} q^{n+1 / 2}\right) .}
\end{aligned}
$$

Setting $d=-(a q)^{1 / 2}$ in Bailey's formula [21, (3.4.1.6)] we find that

$$
\begin{aligned}
& { }_{4} \phi_{3}\left[\begin{array}{cccc}
a, & b, & c, & q^{-n} \\
& a q / b, & a q / c, & b^{2} c^{2} q^{-n-1} / a
\end{array}\right]=\frac{\left(a^{2} q^{2} / b^{2} c^{2},-a^{1 / 2} q^{3 / 2} / b c ; q\right)_{n}}{\left(a q^{2} / b^{2} c^{2},-a^{3 / 2} q^{3 / 2} / b c ; q\right)_{n}} \\
& \cdot{ }_{10} W_{9}\left(-a^{3 / 2} q^{1 / 2} / b c ; a^{1 / 2},-a^{1 / 2},(a q)^{1 / 2}-(a q)^{1 / 2} / b\right. \text {, } \\
& \left.-(a q)^{1 / 2} / c, a^{2} q^{n+2} / b^{2} c^{2}, q^{-n} ; q, q\right)
\end{aligned}
$$

from which, it follows by taking the limit $n \rightarrow \infty$ and replacing $a, b, c$ by $q^{-2 n}$, $-q^{1 / 2-n} e^{i \theta} / a$ and $-q^{1 / 2-n} e^{-i \theta} / a$, respectively, that

$$
\begin{aligned}
& { }_{8} W_{7}\left(-a^{2} q^{-n-1 / 2} ; a e^{i \theta}, a e^{-i \theta}, q^{1 / 2-n},-q^{-n}, q^{-n} ; q,-a^{2} q^{n+1 / 2}\right) \\
& =\frac{\left(a^{4} q^{2 n},-a^{2} q^{1 / 2-n} ; q\right)_{\infty}}{\left(a^{4},-a^{2} q^{n+1 / 2} ; q\right)_{\infty}} \\
& \times_{3} \phi_{2}\left[\begin{array}{cccc}
q^{-2 n}, & -q^{1 / 2-n} e^{i \theta} / a, & -q^{1 / 2-n} e^{-i \theta} / a & \\
& -a q^{1 / 2-n} e^{-i \theta}, & -a q^{1 / 2-n} e^{i \theta} & ; q, a^{4} q^{2 n}
\end{array}\right] .
\end{aligned}
$$

By Sears' transformation formula [20, II(a), p. 173],

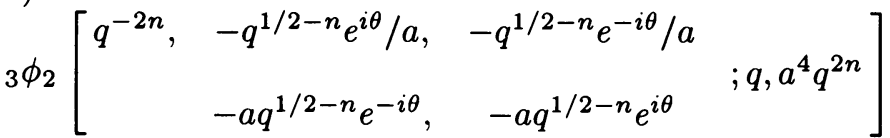

$$
\begin{aligned}
& =\frac{\left(a^{2} ; q\right)_{2 n}}{\left(-a q^{1 / 2-n} e^{-i \theta} ; q\right)_{2 n}}{ }_{3} \phi_{2}\left[\begin{array}{cccc}
q^{-2 n}, & a^{2}, & -q^{1 / 2-n} e^{-i \theta} / a & \\
& q^{1-2 n} / a^{2}, & -a q^{1 / 2-n} e^{i \theta}
\end{array}\right]
\end{aligned}
$$


and hence by combining (3.1), (3.3) and (3.4) we find that (3.5)

$$
p_{n}\left(\cos 2 \theta ; a, a q^{1 / 2},-a,-q^{1 / 2} / a \mid q\right)=\frac{(q ; q)_{2 n}}{\left(a^{4} ; q\right)_{2 n}} \frac{\left(-a^{2} q^{1 / 2} ; q\right)_{n}}{\left(-q^{1 / 2-n} ; q\right)_{n}} G_{2 n}\left(\cos \theta ; a^{2} \mid q\right),
$$

where $G_{n}(x ; \beta \mid q)$ is as defined in (1.25). This is a $q$-analogue of the quadratic transformation formula $[8,10.9(21)]$ :

$$
P_{n}^{(\lambda-1 / 2,-1 / 2)}(\cos 2 \theta)=\frac{(1 / 2)_{n}}{(\lambda)_{n}} C_{2 n}^{\lambda}(\cos \theta)
$$

Similarly one can show that

$$
\begin{aligned}
& p_{n}\left(\cos 2 \theta ; a, a q^{1 / 2},-a q^{1 / 2},-q / a \mid q\right) \\
& \quad=\frac{(q ; q)_{2 n+1}}{\left(a^{4} ; q\right)_{2 n+1}} \frac{\left(-q a^{2} ; q\right)_{n}}{\left(-q^{-n} ; q\right)_{n}} \frac{1+a^{2}}{e^{i \theta}+a e^{-i \theta}} G_{2 n+1}\left(\cos \theta ; a^{2} \mid q\right)
\end{aligned}
$$

which is a $q$-analogue of $[8,10.9(22)]$ :

$$
\cos \theta P_{n}^{(\lambda-1 / 2,1 / 2)}(\cos 2 \theta)=\frac{(1 / 2)_{n+1}}{(\lambda)_{n+1}} C_{2 n+1}^{\lambda}(\cos \theta) .
$$

It is clear from (3.5) and (3.7) that $G_{n}\left(x ; q^{\lambda} \mid q\right)$ behaves like the ultraspherical polynomials $C_{n}^{\lambda}(x)$ in the limit $q \rightarrow 1$, but otherwise quite different from and not nearly as nice as Rogers' continuous $q$-ultraspherical polynomials

$$
C_{n}(\cos \theta ; \beta \mid q)=\sum_{k=0}^{n} \frac{(\beta ; q)_{k}(\beta ; q)_{n-k}}{(q ; q)_{k}(q ; q)_{n-k}} \cos (n-2 k) \theta
$$

The orthogonality and other properties of $C_{n}(x ; \beta \mid q)$ are by now well known, mainly through the works of Askey and Ismail $[4,5]$ and most of these results have proved very useful. We do not expect the functions $G_{n}(x ; \beta \mid q)$ to be half as useful. But the fact that they occur in a $q$-analogue of an important formula, namely (1.4), for ultraspherical polynomials, is probably reason enough to look at them a bit more closely, study their properties and look for other applications. This we plan to do in a later report.

4. A $q$-analogue of the Feldheim-Vilenkin formula. From (1.22) we find that

$$
\begin{aligned}
& \int_{0}^{\pi / 2} \rho\left(\cos 2 \phi ; a, b, c, \mu e^{2 i \theta}, \mu e^{-2 i \theta} \mid q\right) \frac{\left(a b c \mu e^{2 i \theta}, a b c \mu e^{-2 i \theta} ; q\right)_{n}}{\left(a b c \mu^{2} e^{2 i \phi}, a b c \mu^{2} e^{-2 i \phi} ; q\right)_{n}} \\
& \text { - } p_{n}(\cos 2 \phi ; a, b, c, d \mid q) d \phi \\
& =\frac{1}{2} g\left(a, b, c, \mu e^{2 i \theta}, \mu e^{-2 i \theta} \mid q\right) \frac{(b c ; q)_{n}}{\left(b c \mu^{2} ; q\right)_{n}} p_{n}\left(\cos 2 \theta ; a \mu, b \mu, c \mu, d \mu^{-1} \mid q\right) \text {, }
\end{aligned}
$$

where

$$
\begin{aligned}
& \rho\left(\cos 2 \phi ; a_{1}, a_{2}, a_{3}, a_{4}, a_{5} \mid q\right) \\
& =\frac{h(\cos 2 \phi, 1) h(\cos 2 \phi,-1) h\left(\cos 2 \phi, q^{1 / 2}\right) h\left(\cos 2 \phi,-q^{1 / 2}\right) h\left(\cos 2 \phi, a_{1} a_{2} a_{3} a_{4} a_{5}\right)}{h\left(\cos 2 \phi, a_{1}\right) h\left(\cos 2 \phi, a_{2}\right) h\left(\cos 2 \phi, a_{3}\right) h\left(\cos 2 \phi, a_{4}\right) h\left(\cos 2 \phi, a_{5}\right)} .
\end{aligned}
$$


Using (3.5) we then have

$$
\begin{gathered}
\int_{0}^{\pi / 2} \rho\left(\cos 2 \phi ; a, a q^{1 / 2},-a, \mu e^{2 i \theta}, \mu e^{-2 i \theta} \mid q\right) \frac{\left(-q^{1 / 2} a^{3} \mu e^{2 i \theta},-q^{1 / 2} q^{3} \mu e^{-2 i \theta} ; q\right)_{n}}{\left(-q^{1 / 2} a^{3} \mu^{2} e^{2 i \phi},-q^{1 / 2} a^{3} \mu^{2} e^{-2 i \phi} ; q\right)_{n}} \\
\cdot G_{2 n}\left(\cos \phi ; a^{2} \mid q\right) d \phi \\
=\frac{1}{2} g\left(a, a q^{1 / 2},-a, \mu e^{2 i \theta}, \mu e^{-2 i \theta} \mid q\right) \frac{\left(a^{4} ; q\right)_{2 n}}{\left(a^{4} \mu^{4} ; q\right)_{2 n}} G_{2 n}\left(\cos \theta ; a^{2} \mu^{2} \mid q\right)
\end{gathered}
$$

Similarly, the use of (3.7) gives

$$
\begin{array}{r}
\int_{0}^{\pi / 2} \rho\left(\cos 2 \phi ; a, a q^{1 / 2},-a q^{1 / 2}, \mu e^{2 i \theta}, \mu e^{-2 i \theta} \mid q\right) \frac{\left(-q a^{3} \mu e^{2 i \theta},-q a^{3} \mu e^{-2 i \theta} ; q\right)_{n}}{\left(-q a^{3} \mu^{2} e^{2 i \phi},-q a^{3} \mu^{2} e^{-2 i \phi} ; q\right)_{n}} \\
\cdot \frac{e^{i \theta}+\mu a e^{-i \theta}}{e^{i \phi}+a e^{-i \phi}} G_{2 n+1}\left(\cos \phi ; a^{2} \mid q\right) d \phi \\
=\frac{1}{2} g\left(a, a q^{1 / 2},-a q^{1 / 2}, \mu e^{2 i \theta}, \mu e^{-2 i \theta} \mid q\right) \frac{1+a^{2} \mu^{2}}{1+a^{2}} \frac{\left(a^{4} ; q\right)_{2 n+1}}{\left(a^{4} \mu^{4} ; q\right)_{2 n+1}} \\
\cdot G_{2 n+1}\left(\cos \theta ; a^{2} \mu^{2} \mid q\right) .
\end{array}
$$

Combining (4.3) and (4.4) we get the formula

$$
\begin{array}{rl}
\int_{0}^{\pi / 2} & K\left(\cos \theta, \cos \phi ; a^{2} \mid q\right) \frac{h\left(\cos 2 \phi,-a^{3} \mu^{2} q^{(n+1) / 2}\right)}{h\left(\cos 2 \theta,-a^{3} \mu q^{(n+1) / 2}\right)} \\
& \cdot \frac{\left(-a q^{(n+1) / 2-[n / 2]} e^{-2 i \phi},-a q^{(1-n) / 2+[n / 2]} e^{2 i \phi} ; q\right)_{\infty}}{\left(-a \mu q^{(n+1) / 2-[n / 2]} e^{-2 i \theta},-a \mu q^{(1-n) / 2+[n / 2]} e^{2 i \theta} ; q\right)_{\infty}} \\
= & e^{2 i(\theta-\phi)(n / 2-[n / 2])} \cdot G_{n}\left(\cos \phi ; a^{2} \mid q\right) d \phi \\
\left(a^{4} \mu^{4} ; q\right)_{n} & a_{n}\left(\cos \theta ; a^{2} \mu^{2} \mid q\right),
\end{array}
$$

where

$$
\begin{aligned}
& K\left(\cos \theta, \cos \phi ; a^{2} \mid q\right)=\frac{\left(q, a^{4}, \mu^{2}, a^{2} \mu^{2} ; q\right)_{\infty}}{\pi\left(a^{4} \mu^{4}, a^{2} ; q\right)_{\infty}} \\
& \cdot \frac{h(\cos 4 \phi, 1) h\left(\cos 4 \theta, a^{2} \mu^{2}\right)}{h\left(\cos 4 \phi, a^{2}\right) h\left(\cos 2 \phi, \mu e^{2 i \theta}\right) h\left(\cos 2 \phi, \mu e^{-2 i \theta}\right)}, \quad|a|<1,0<\mu<1 .
\end{aligned}
$$

Replacing $a, \mu$ by $q^{\lambda / 2}, q^{(\nu-\lambda) / 2}, \nu>\lambda>-1 / 2$, it can be shown by using the $q$-gamma function

$$
\Gamma_{q}(x)=\frac{(q ; q)_{\infty}}{\left(q^{x} ; q\right)_{\infty}}(1-q)^{1-x}, \quad \Gamma(x)=\lim _{q \rightarrow 1} \Gamma_{q}(x)
$$

that (4.5) reduces to

$$
\begin{aligned}
& \frac{C_{n}^{\nu}(\cos \theta)}{C_{n}^{\nu}(1)} \frac{\sin ^{2 \nu-1} \theta}{\cos ^{n+2 \lambda+1} \theta} \\
& \quad=\frac{2 \Gamma(\nu+1 / 2)}{\Gamma(\lambda+1 / 2) \Gamma(\nu-\lambda)} \int_{0}^{\theta} \sin ^{2 \lambda} \phi \frac{\left[\cos ^{2} \phi-\cos ^{2} \theta\right]^{\nu-\lambda-1}}{\cos ^{n+2 \nu} \phi} \frac{C_{n}^{\lambda}(\cos \phi)}{C_{n}^{\lambda}(1)} d \phi
\end{aligned}
$$

which is equivalent to Feldheim-Vilenkin formula (1.4); see Askey [1, pp. 23-24]. The evaluation of the limit of $K(\cos \theta, \cos \phi ; \mid q)$ as $q \rightarrow 1$ is a bit tricky, but similar calculations were done in Nassrallah and Rahman [16]. 


\section{REFERENCES}

1. R. Askey, Orthogonal polynomials and special functions, Regional Conf. Series in Appl. Math., SIAM, Philadelphia, Pa., 1975.

2. __ Jacobi polynomials I. New proofs of Koornwinder's Laplace-type integral representations and Bateman's bilinear sum, SIAM J. Math. Anal. 5 (1974), 119-124.

3. R. Askey and J. Fitch, Integral representations for Jacobi polynomials and some applications, J. Math. Anal. Appl. 26 (1969), 411-437.

4. R. Askey and M. E. H. Ismail, A generalization of ultraspherical polynomials, Studies in Pure Mathematics (P. Erdös, ed.), Birkhauser, Boston, Mass., 1982, pp. 56-78.

5. _ The Rogers q-ultraspherical polynomials, Approximation Theory. III (E. W. Cheney, ed.), Academic Press, New York, 1980, pp. 175-182.

6. R. Askey and J. Wilson, Some basic hypergeometric orthogonal polynomials that generalize Jacobi polynomials, Mem. Amer. Math. Soc., no 319 (1985).

7. W. N. Bailey, Generalized hypergeometric series, Stechert-Hafner, New York and London, 1964.

8. A. Erdélyi, et al., Higher transcendental functions, vol. 2, McGraw-Hill, New York, 1953.

9. L. Fejér, Sur le développement d'une fonction arbitraire suivant les fonctions de Laplace, C. R. Acad. Sci. Paris 146 (1908), 224-225; reproduced in Gesammelte Arbeiten I. 319-322.

10. E. Feldheim, On the positivity of certain sums of ultraspherical polynomials, J. Analyse Math. 11 (1963), 275-284.

11. G. Gasper and M. Rahman, Positivity of the Poisson kernel for the continuous q-Jacobi polynomials and some quadratic transformation formulas for basic hypergeometric series, SIAM J. Math. Anal. 17 (1986), 970-999.

12. T. Koornwinder, The addition formula for Jacobi polynomials. I, Summary of results, Indag. Math. 34 (1972), 293-316.

13. _ Jacobi polynomials. II, An analytic proof of the product formula, SIAM J. Math. Anal. 5 (1974), 125-137.

14. _ Jacobi polynomials. III, An analytic proof of the addition formula, SIAM J. Math. Anal. 6 (1975), 533-543.

15. T. P. Laine, Projection formulas and a new proof of the addition formula for the Jacobi polynomials, SIAM J. Math. Anal. 13 (1982), 324-330.

16. B. Nassrallah and M. Rahman, Projection formulas, a reproducing kernel and a generating function for $q$-Wilson polynomials, SIAM J. Math. Anal. 16 (1985), 186-197.

17. M. Rahman, An integral representation of $a_{10} \phi_{9}$ and continuous biorthogonal ${ }_{10} \phi_{9}$ rational functions, Canad. J. Math. 38 (1986), 605-618.

18. _ The linearization of the product of continuous $q$-Jacobi polynomials, Canad. J. Math. 33 (1981), 961-987.

19. (1986), 309-322.

20. D. B. Sears, On the transformation theory of basic hypergeometric functions, Proc. London Math. Soc. (2) 53 (1951), 158-180.

21. L. J. Slater, Generalized hypergeometric functions, Cambridge Univ. Press, 1966.

22. G. Szegö, Orthogonal polynomials (4th ed.), Amer. Math. Soc. Colloq. Publ., Vol. 23, Amer. Math. Soc., Providence, R.I., 1975.

23. N. J. Vilenkin, Some relations for Gegenbauer functions, Uspekhi Mat. Nauk (N.S.) 13 (1958), no. 3(81), 167-172 (Russian).

Department of Mathematics and Statistics, Carleton University, Ottawa, ONTARIO K1S 5B6, CANADA 